\title{
Synaptic Plasticity Associated with a Memory Engram in the Basolateral Amygdala
}

\author{
Ayako Nonaka, ${ }^{1 \star}$ Takeshi Toyoda, ${ }^{1 \star}$ Yuki Miura, ${ }^{1}$ Natsuko Hitora-Imamura, ${ }^{1}$ Masamitsu Naka, ${ }^{1}$ Megumi Eguchi, ${ }^{2}$ \\ Shun Yamaguchi, ${ }^{2,3}$ Yuji Ikegaya, ${ }^{1,4}$ Norio Matsuki, ${ }^{1}$ and Hiroshi Nomura ${ }^{1}$ \\ ${ }^{1}$ Laboratory of Chemical Pharmacology, Graduate School of Pharmaceutical Sciences, The University of Tokyo, Tokyo 113-0033, Japan, ${ }^{2}$ Division of \\ Morphological Neuroscience, Gifu University Graduate School of Medicine, Gifu 501-1194, Japan, ${ }^{3}$ PRESTO, Japan Science and Technology Agency (JST), \\ Saitama 332-0012, Japan, and ${ }^{4}$ Center for Information and Neural Networks, Suita City, Osaka 565-0871, Japan
}

Synaptic plasticity is a cellular mechanism putatively underlying learning and memory. However, it is unclear whether learning induces synaptic modification globally or only in a subset of neurons in associated brain regions. In this study, we genetically identified neurons activated during contextual fear learning and separately recorded synaptic efficacy from recruited and nonrecruited neurons in the mouse basolateral amygdala (BLA). We found that the fear learning induces presynaptic potentiation, which was reflected by an increase in the miniature EPSC frequency and by a decrease in the paired-pulse ratio. Changes occurred only in the cortical synapses targeting the BLA neurons that were recruited into the fear memory trace. Furthermore, we found that fear learning reorganizes the neuronal ensemble responsive to the conditioning context in conjunction with the synaptic plasticity. In particular, the neuronal activity during learning was associated with the neuronal recruitment into the context-responsive ensemble. These findings suggest that synaptic plasticity in a subset of BLA neurons contributes to fear memory expression through ensemble reorganization.

\section{Introduction}

One cellular mechanism underlying fear conditioning is likely to be synaptic plasticity in the amygdala. Fear conditioning potentiates synaptic strength in the amygdala (McKernan and Shinnick-Gallagher, 1997; Tsvetkov et al., 2002) and is impaired when synaptic plasticity in the amygdala is inhibited (Fanselow and Kim, 1994; Rumpel et al., 2005). However, it is unclear whether fear learning induces synaptic modification in the amygdala globally or only in a subset of neurons. Because studies using activity-dependent gene expression showed that a subset of amygdala neurons are involved in fear conditioning (Han et al., 2007, 2009; Reijmers et al., 2007), we hypothesized that fear learning induces synaptic modification only in neurons that are recruited into the memory trace. Furthermore, if this is the case, synaptic modification in a subset of neurons would likely lead to a reorganization of amygdala neuronal ensemble activity.

Received Sept. 25, 2013; revised May 20, 2014; accepted June 5, 2014.

Author contributions: Y.I., N.M., and H.N. designed research; A.N., T.T., Y.M., N.H.-I., M.N., and H.N. performed research; M.E. and S.Y. contributed unpublished reagents/analytic tools; A.N., T.T., Y.M., and H.N. analyzed data; A.N. and H.N. wrote the paper.

This work was supported by a Grant-in-Aid for Young Scientists (B) (25830002 to H.N.); Grant-in-Aid for Scientific Research on Innovative Areas, "Mesoscopic Neurocircuitry" (23115101 to H.N.); "The Science of Mental Time" (26119507 to H.N. and 25119004 to Y.I.); and "Memory Dynamism" (26115509 to H.N.). We thank Dr. Hiroyuki Hioki (Kyoto University) for technical advice in in situ hybridization and immunohistochemistry, Dr. Paul F Worley (Johns Hopkins University) for the Arc and Homer $1 a$ cDNA used in this study, and the University of Tokyo/Leica microsystems imaging center for their assistance in obtaining the imaging data.

${ }^{*}$ A.N. and T.T. contributed equally to this work.

The authors declare no competing financial interests.

Correspondence should be addressed to Hiroshi Nomura, PhD, Laboratory of Chemical Pharmacology, Graduate School of Pharmaceutical Sciences, The University of Tokyo 7-3-1 Hongo, Bunkyo-ku, Tokyo 113-0033, Japan. E-mail: nomura@mol.f.u-tokyo.ac.jp.

DOI:10.1523/JNEUROSCI.4233-13.2014

Copyright $\odot 2014$ the authors $\quad 0270-6474 / 14 / 349305-05 \$ 15.00 / 0$
Here, we tested these ideas by combining whole-cell recordings with imaging of neuronal activity histories. We examined synaptic plasticity and ensemble reorganization associated with contextual fear conditioning. Further, we focused our analysis on the basolateral amygdala (BLA) because of its importance in contextual fear conditioning (Maren, 2001).

\section{Materials and Methods}

Animals. All experiments were approved by the animal experiment ethics committee at the University of Tokyo (approval number 24-8, 24-10), and were in accordance with the University of Tokyo guidelines for the care and use of laboratory animals. For catFISH, we used male C57BL/6J mice ( $8-15$ weeks old; SLC) on a $12 \mathrm{~h}$ light/dark cycle with lights on from 7:00 A.M. to 7:00 P.M., with access to food and water ad libitum. For electrophysiological experiments, we used male or female Arc-dVenus transgenic mice (3-5 weeks old; Eguchi and Yamaguchi, 2009).

Behavioral procedures. In the electrophysiological experiments with fear memory retrieval (Fig. 1), we examined synaptic efficacy in the neurons that were activated during memory retrieval. On day 1 , mice in the fear conditioning (FC) group were conditioned in a conditioning chamber with three footshocks ( $1 \mathrm{~mA}, 2 \mathrm{~s}, 150 \mathrm{~s}$ intervals). Mice in the immediate shock (IS) group received a footshock $(1 \mathrm{~mA}, 6 \mathrm{~s})$ immediately after they were placed in the chamber. On day 2 , both groups were re-exposed to the chamber for $5 \mathrm{~min}$ and killed $5 \mathrm{~h}$ later, when dVenus fluorescence reaches a plateau (Eguchi and Yamaguchi, 2009). In the electrophysiological experiments without fear memory retrieval (Fig. 2), we examined synaptic efficacy in the neurons that were activated during fear conditioning. Mice were conditioned in the conditioning chamber with three footshocks and killed $5 \mathrm{~h}$ later.

In the catFISH experiments that examined fear conditioning-induced ensemble reorganization, the behavioral test comprised three sessions. In session 1, mice in the FC group were placed in the conditioning chamber for $5 \mathrm{~min}$. The mice were returned to their home cage and $36 \mathrm{~min}$ later, in session 2 , they were conditioned with three footshocks $(1 \mathrm{~mA}, 2 \mathrm{~s}, 150 \mathrm{~s}$ 
intervals) in the chamber. They were returned to their home cage again, and 20 min later (session 3) they were re-exposed to the chamber for 5 min to induce recall of the fear memory. The IS group, a control for associative learning, was given footshocks 0,10 , and $20 \mathrm{~s}$ after being placed in the chamber in session 2, instead of the fear conditioning protocol. In the catFISH experiments that examined the time course of the cytoplasmic Homer 1a signals, mice were exposed to the conditioning chamber for $5 \mathrm{~min}$ without shock. They were killed immediately, $25 \mathrm{~min}$ or $70 \mathrm{~min}$ after the context exposure.

All sessions were video recorded to enable the automatic scoring of freezing behavior according to the previously described method (Nomura and Matsuki, 2008).

Electrophysiology. Mice were deeply anesthetized with diethyl ether and decapitated $5 \mathrm{~h}$ after re-exposure to the conditioning context. Brains were removed quickly, and coronal slices ( $300 \mu \mathrm{m}$ thick) containing the BLA were prepared with a vibratome (VT 1200S; Leica) in ice-cold, oxygenated $\left(95 \% \mathrm{O}_{2} / 5 \% \mathrm{CO}_{2}\right)$ modified artificial CSF (mACSF) containing $222.1 \mathrm{~mm}$ sucrose, $27 \mathrm{~mm} \mathrm{NaHCO} 3,1.4 \mathrm{~mm} \mathrm{NaH}_{2} \mathrm{PO}_{4}, 2.5 \mathrm{~mm} \mathrm{KCl}$, $0.5 \mathrm{~mm}$ ascorbic acid, $1 \mathrm{~mm} \mathrm{CaCl}_{2}$, and $7 \mathrm{~mm} \mathrm{MgSO}_{4}$.

Picrotoxin $(100 \mu \mathrm{M})$ was added to ACSF $(127 \mathrm{~mm} \mathrm{NaCl}, 1.6 \mathrm{~mm} \mathrm{KCl}$, $1.24 \mathrm{~mm} \mathrm{KH}_{2} \mathrm{PO}_{4}, 1.3 \mathrm{~mm} \mathrm{MgSO}_{4}, 2.4 \mathrm{~mm} \mathrm{CaCl}_{2}, 26 \mathrm{~mm} \mathrm{NaHCO}_{3}$, and $10 \mathrm{~mm}$ glucose). Whole-cell patch-clamp recordings were performed with glass microelectrodes (3-8 $\mathrm{M} \Omega$ ) filled with internal solution (135 $\mathrm{mm}$ K-gluconate, $4 \mathrm{~mm} \mathrm{KCl}, 10 \mathrm{~mm}$ phosphocreatine- $\mathrm{Na}_{2}, 10 \mathrm{~mm}$ HEPES, 4 mm MgATP, and 0.3 mм Na $\mathrm{NTP}_{2} \mathrm{pH}$ 7.2-7.3, 280-295 $\mathrm{mOsm})$. Because the BLA receives projections from diverse cortices and thalamus including the entorhinal and perirhinal cortices and mediodorsal thalamic nucleus, which are involved in contextual fear conditioning (Maren and Fanselow, 1997; Sacchetti et al., 1999; Li et al., 2004), we examined the effect of contextual fear conditioning on the cortico-BLA and thalamo-BLA synapses. Electrical stimulation was applied to the internal capsule to evoke EPSCs in BLA neurons from thalamic afferents, or to the external capsule to evoke EPSCs from cortical afferents, using bipolar tungsten electrodes $(0.1-1 \mathrm{M} \Omega)$. Paired stimuli were given with an interstimulus interval of $50 \mathrm{~ms}$, and the ratio between the amplitude of the second and first EPSCs was calculated. Miniature EPSCs (mEPSCs) were recorded at a holding potential of $-70 \mathrm{mV}$ in the presence of tetrodotoxin $(1 \mu \mathrm{M})$. mEPSCs were detected using an in-house MATLAB program and were defined as inward currents with amplitudes $>7 \mathrm{pA}$ unless stated (Miura et al., 2012). Data were sampled at $20 \mathrm{kHz}$ and filtered at $2 \mathrm{kHz}$ using an Axopatch 200B, 700B amplifier (Molecular Devices), DIGIDATA1320A, 1440A (Molecular Devices), and pClamp 10.2 (Molecular Devices). All data were acquired, stored, and analyzed using Clampex 10, Clampfit, and MATLAB.

Fluorescence in situ hybridization. Mice were killed immediately after session 3, and their brains were removed and frozen quickly. In situ hybridization was performed according to previously published protocols (Nomura et al., 2012). The coronal brain sections $(20 \mu \mathrm{m})$ were hybridized with the riboprobes (DIG-Homer $1 a$ antisense riboprobe, 2 $\mu \mathrm{g} / \mathrm{ml}$; Fluorescein-Arc antisense riboprobe, $1 \mu \mathrm{g} / \mathrm{ml}$ ). The signals were detected with an anti-Fluorescein HRP-conjugated antibody (1:200; PerkinElmer); a TSA Plus DNP System (1:10; PerkinElmer); an anti-DIG peroxidase-conjugated antibody (1:500; Roche); Tyramide-biotin (1: 5000), an Alexa488-conjugated anti-DNP antibody (Invitrogen); and Alexa594-conjugated streptavidin (Invitrogen). The nuclei were counterstained with Hoechst.

$Z$-stacks of 1- $\mu \mathrm{m}$-thick optical sections were acquired with LSM-510 (Zeiss) and CV1000 (Yokogawa Denki) confocal microscopes using $40 \times$ objective lenses. Only cells that were presumptive neurons with whole, large nuclei stained diffusely with the Hoechst dye were included in the analysis. The designation "intranuclear positive" was assigned to neurons that exhibited one or two of the characteristic intense intranuclear areas of fluorescence. The designation "cytoplasmic positive" was assigned to neurons that contained perinuclear/cytoplasmic labeling over multiple optical sections.

Immunohistochemistry. For dVenus detection, mice were transcardially perfused with $4 \%$ PFA in PBS $5 \mathrm{~h}$ after re-exposure to the conditioning context. The sections were incubated with rabbit anti-GFP antibody
(1:1000; Invitrogen), Alexa488 anti-rabbit IgG (1:500; Invitrogen), and NeuroTrace Blue (1:50; Invitrogen).

Data analysis. All values are given as the mean \pm SEM. Two-way factorial ANOVA, repeated-measure ANOVA, Student's $t$ test, the Tukey-Kramer test, and the paired $t$ test were used for appropriate comparisons.

An overlap score between neuronal populations that were active during both sessions 1 and 3 was obtained as follows: $S_{1}=$ percentage of total cytoplasmic Homer $1 a+$ neurons, $S_{3}=$ percentage of total nuclear Arc + neurons, $\mathrm{S}_{13}=$ percentage of neurons in which both cytoplasmic Homer $1 a$ and nuclear Arc were observed, chance level $\left(\mathrm{C}_{13}\right)=\mathrm{S}_{1} \times \mathrm{S}_{3} / 100$, overlap score between sessions 1 and $3=\left(S_{13}-C_{13}\right) /\left(S_{3}-C_{13}\right) \times 100$. The recruiting score was calculated as the percentage of neurons that changed their activity from inactive in session 1 to active in session 3 .

\section{Results}

\section{Contextual fear conditioning induces presynaptic potentiation in BLA neurons recruited into a fear memory trace}

To identify the neurons that were recruited into a memory trace, we used $A r c-d V e n u s$ transgenic mice. These mice express a destabilized version of the fluorescent protein Venus under the control of the Arc promoter (Eguchi and Yamaguchi, 2009). The Arc$d$ Venus mice allowed us to identify the neurons that were recruited into the memory trace by dVenus fluorescence produced by Arc activation during fear memory expression. The mice received contextual fear conditioning, and $24 \mathrm{~h}$ later they were re-exposed to the conditioning context (Fig. 1A). They showed robust freezing during the re-exposure to the context (Fig. $1 B$ ), suggesting that they recalled the contextual fear memory. They were killed $5 \mathrm{~h}$ after the context re-exposure for the subsequent electrophysiological studies. An IS group, which received a footshock immediately after they were placed in the chamber, was prepared as a control for associative fear learning (Fig. 1B). The percentage of $\mathrm{dVenus}+\mathrm{BLA}$ neurons in $\mathrm{FC}$ mice was higher than that of home cage (HC) controls (Fig. 1C,D).

Brain slices were prepared for whole-cell recordings from BLA neurons. We measured mEPSCs from dVenus $+(\mathrm{V}+)$ and $\mathrm{dVe}-$ nus $-(\mathrm{V}-)$ neurons of the mice in the FC and IS groups. The mEPSC frequency of the dVenus + neurons in the FC group was higher than that of the dVenus - neurons in the FC group and the dVenus + neurons in the IS group (Fig. $1 E, F$ ). There was no significant group $\times$ dVenus interaction for mEPSC amplitude (Fig. $1 G$ ).

To probe the synaptic efficacy of dVenus+ neurons in the FC group, we measured the evoked EPSCs and paired-pulse ratio (PPR). The EPSC amplitude of the dVenus+ neurons in the FC group was higher than that of the dVenus - neurons in the FC group in the cortical, but not the thalamic, pathway (Fig. $1 H, I$ ). PPR in the $\mathrm{dVenus}+$ neurons of the FC group was lower than that in dVenusneurons of the FC group in the cortical, but not the thalamic, pathway (Fig. $1 \mathrm{~J}, K$ ). These results indicate that the synaptic efficacy of cortico-amygdala synapses is presynaptically enhanced in the FC group, and that this enhancement is restricted to neurons that were recruited into the memory trace (i.e., dVenus + neurons).

We tested whether fear memory retrieval is required for the high mEPSC frequency in the recruited neurons. Arc-dVenus mice were killed $5 \mathrm{~h}$ after they received fear conditioning without re-exposure to the conditioning context (Fig. 2A). BLA dVenus+ neurons showed higher mEPSC frequency and amplitude than dVenus - neurons (Fig. $2 B-D$ ). These results suggest that the high $\mathrm{mEPSC}$ frequency in the recruited neurons is attributable to fear conditioning.

In all the analyses described above, we detected mEPSCs with a 7 pA threshold. This analysis strictly removed noise (false positive EPSCs) but could miss small mEPSCs. To avoid missing 
A Arc-dVenus mice

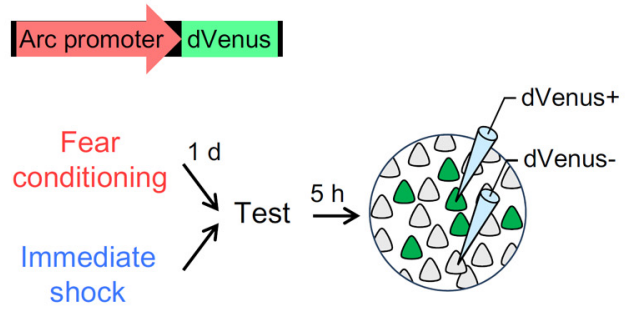

B

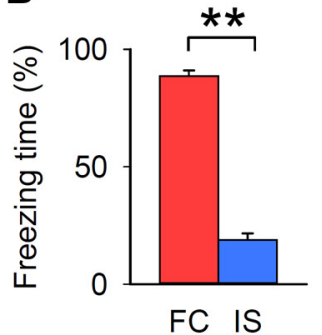

C

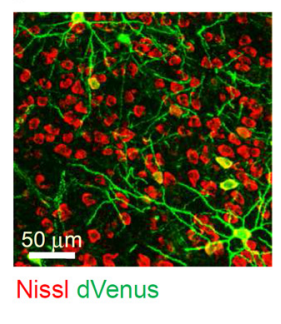

D

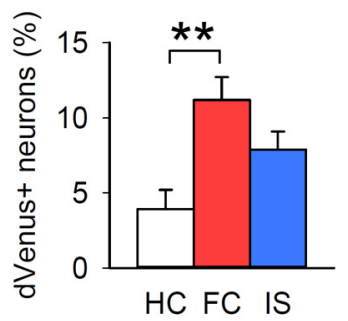

E

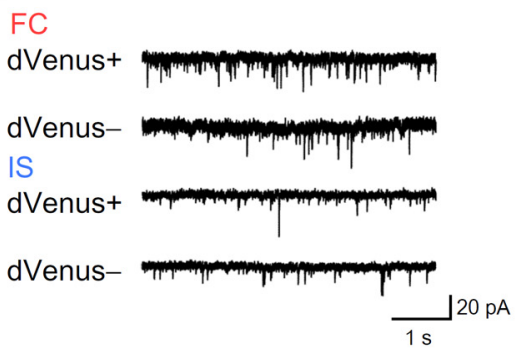

F

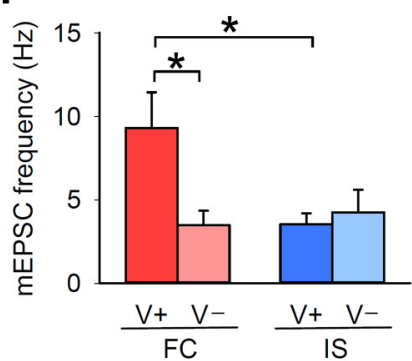

G

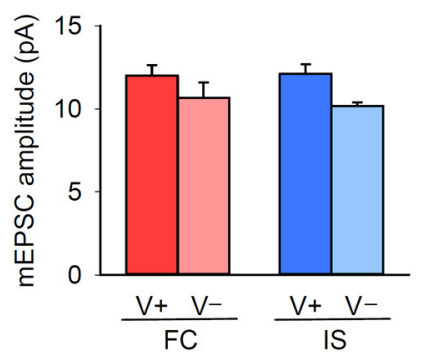

H

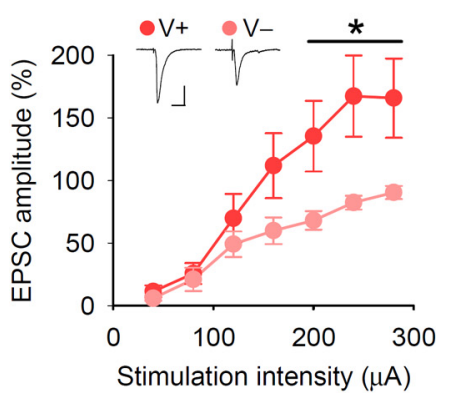

I

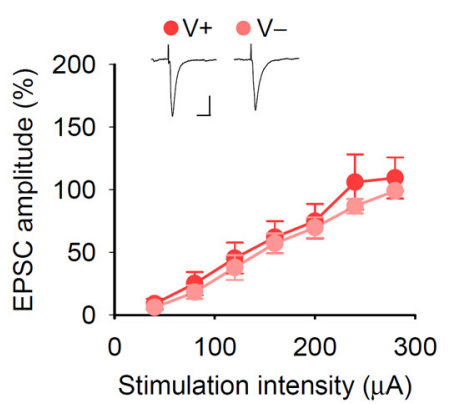

J Cortical pathway

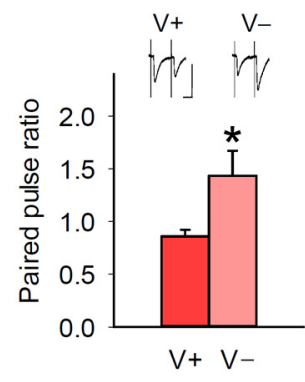

K Thalamic pathway

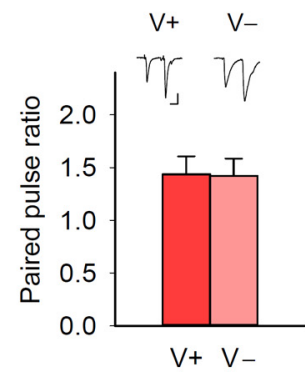

Figure 1. Contextual fear conditioning induces presynaptic potentiation in BLA neurons recruited into a fear memory trace. $\boldsymbol{A}$, Experimental procedure. $\boldsymbol{B}$, The $\mathrm{FC}$ group showed longer freezing time during the test than the IS group. $C$, A representative fluorescence image of $B L A$ neurons with dVenus. $D$, Re-exposure to the conditioning context increased the proportion of dVenus + neurons in the BLA (one-way ANOVA, $F_{(2,18)}=6.7, p=0.0068$; Tukey's test, home cage $(H C)$ vs $\left.F C, p=0.0050\right) . E, F$, dVenus $+(V+$ ) neurons of the FC group had a higher mEPSC frequency than dVenus $(V-)$ neurons of the $F C$ group and dVenus + neurons of the IS group $(n=10 \mathrm{dVenus}+$ and $10 \mathrm{dVenus}$ - neurons per behavioral group from 14 mice in the $\mathrm{FC}$ group and 12 mice in the IS group; two-way ANOVA, $F_{(1,36)}=4.9, p=0.033$; dVenus $+(\mathrm{FC})$ vs dVenus $-(\mathrm{FC}), p=0.042 ; \mathrm{dVenus}+(\mathrm{FC})$ vs dVenus $\left.+(\mathrm{IS}), p=0.024\right)$. $G$, There was no significant group $\times$ dVenus interaction of $\mathrm{mEPSC}$ amplitude $\left(F_{(1,36)}=0.33, p=0.57\right) . \boldsymbol{H}, \boldsymbol{I}$, The EPSC amplitude of the dVenus + neurons in the FC group was higher than that of the dVenus - neurons in the $\mathrm{FC}$ group in the cortical but not thalamic pathway $\left(n=7-10\right.$ neurons from 5 mice; cortical pathway, repeated-measures ANOVA, $\left.F_{(6,108)}=4.5, p=4.1 \times 10^{-4}\right)$. Calibration: $20 \mathrm{~ms}, 100 \mathrm{pA} . J, K$, The PPR in dVenus + neurons in the FC group was lower than that of dVenus - neurons in the FC group in the cortical but not thalamic pathway ( $n=7-11$ neurons from 7 mice; cortical pathway, Student's $t$ test, $t_{(11)}=2.3$, $p=0.041)$. Calibration: $20 \mathrm{~ms}, 50 \mathrm{pA} .{ }^{* *} p<0.01,{ }^{*} p<0.05$.

small mEPSCs, we also performed an additional analysis detecting mEPSCs with a $5 \mathrm{pA}$ threshold. Similarly with the $7 \mathrm{pA}$ threshold analyses, we found that BLA dVenus+ neurons showed a higher mEPSC frequency (dVenus $+: 12.1 \pm 2.8 \mathrm{~Hz}$; dVenus-: $6.9 \pm 2.1 \mathrm{~Hz}$; paired $t$ test, $\left.t_{(5)}=4.7, p=0.0051\right)$ and amplitude (dVenus+: $10.0 \pm 0.19 \mathrm{pA}$; dVenus -: $8.3 \pm 0.14$; $\left.t_{(5)}=9.9, p=0.0002\right)$ than $\mathrm{dVenus}-$ neurons.

Fear conditioning reorganizes a context-responsive BLA neuronal ensemble based on the activity of individual BLA neurons during fear conditioning

Because synaptic potentiation in a subset of neurons is likely to lead to a reorganization of neuronal ensembles, we tested whether a BLA neuronal ensemble responsive to context is altered by fear conditioning. Mice in the FC group were subjected to context exposure in session 1 (S1), contextual fear conditioning in session 2 (S2), and context re-exposure in session 3 (S3) with 36 and 20 min intervals (Fig. 3A). They spent a much greater time freezing in session 3 versus session 1 (Fig. $3 B$ ). The IS group demonstrated significantly less freezing in session 3 than the FC group.

To identify neurons that were active during each session, we used temporal activity mapping with cellular resolution by observing Arc and Homer 1a RNA (i.e., catFISH; Guzowski et al., 1999; Marrone et al., 2008). Transcribed Arc RNA first appears in neuronal nuclei, after which processed $\operatorname{Arc}$ mRNA accumulates in the cytoplasm. Homer $1 a$ RNA is transcribed later than Arc RNA. Previously, we reported that nuclear Arc is observed immediately after neuronal activity and that cytoplasmic Arc and nuclear Homer $1 a$ are observed 25-30 min after neuronal activity in the amygdala (Hashikawa et al., 2011; Nomura et al., 2012). Because the characteristics of the cytoplasmic Homer 1a signal have been described for hippocampal neurons (Marrone et al., 2008) but not amygdala neurons, we first examined the time course of the cytoplasmic Homer $1 a$ signal after neuronal activity. We found that in the basolateral amygdala, as well as the hippocampal CA1, more cytoplasmic Homer $1 a+$ neurons were observed when mice were killed 70 min after context exposure compared 
A

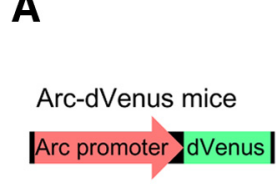

B

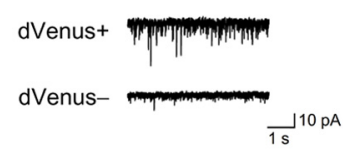

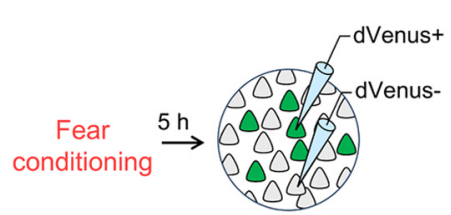

C

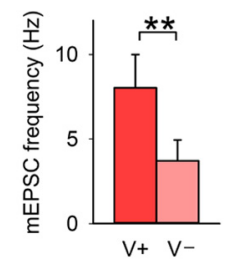

D

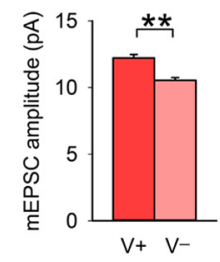

Figure 2. Enhanced synaptic efficacy in the BLA neurons recruited into a fear memory trace is due to fear conditioning but not fear memory retrieval. $A$, Experimental procedure. $B$, Representative $m E P S C$ traces recorded from dVenus + and dVenus - neurons. $\boldsymbol{C}, \boldsymbol{D}$, dVenus + neurons had a higher mEPSC frequency $(\boldsymbol{C})$ and amplitude $(\boldsymbol{D})$ than dVenus - neurons $(n=6$ $\mathrm{dVenus}+$ and $6 \mathrm{dVenus}-$ neurons from 5 mice; frequency, paired $t$ test, $t_{(5)}=5.4, p=$ 0.0029 ; amplitude, $\left.t_{(5)}=7.8, p=0.00056\right) ;{ }^{* *} p<0.01$.

\section{A}

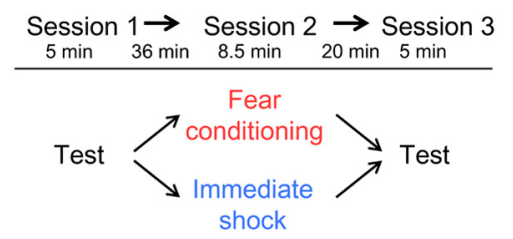

C

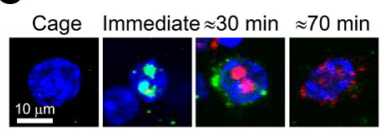

Hoechst Arc Homer 1 a

D

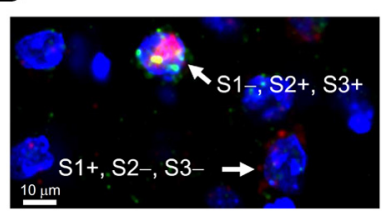

B

E

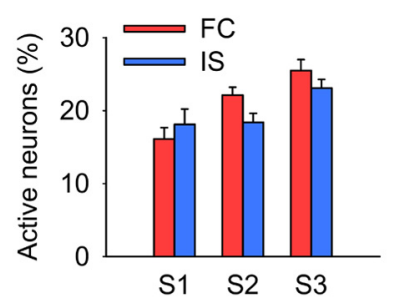

Figure 3. Large-scale activity imaging before, during, and after fear conditioning in the BLA. $\boldsymbol{A}$, Experimental procedure ( $n=8$ mice per group). $\boldsymbol{B}$, The $\mathrm{FC}$ but not the IS group exhibited contextual conditioned fear in session 3 (repeated-measures ANOVA, $F_{(1,14)}=23.8, p=$ 0.00025 ; FC vs IS in session $3, p=0.00017$ ). $C$, Representative images of individual $B L A$ neurons from fluorescent in situ hybridization of $\operatorname{Arc}$ and Homer $1 a . D$, A representative image of $\operatorname{Arc}$ and Homer $1 a$ RNA expression from the FC group. A neuron with nuclear and cytoplasmic Arc and nuclear Homer $1 a$ was activated in sessions 2 and $3(\mathrm{~S} 1-, \mathrm{S} 2+, \mathrm{S} 3+)$. A neuron with only cytoplasmic Homer $1 a$ was activated in session $1(\mathrm{~S} 1+, \mathrm{S} 2-, \mathrm{S} 3-)$. E, Percentage of BLA neurons active in each session was comparable between the $\mathrm{FC}$ and IS groups $(n=319.0 \pm$ 18.1 neurons in $\mathrm{F}$ (group and $313.3 \pm 17.6$ neurons in IS group per mouse).

with 0 and $25 \min$ ( $p=0.00052$ and 0.0013 , respectively). Therefore, in the following analysis, we identified neurons that were activated during session 1 ( $\sim 70$ min before being killed), session 2 ( $\sim 30$ min before being killed), and session 3 (immediately before being killed) based on the "cytoplasmic Homer 1a," "nuclear Homer $1 a$ and cytoplasmic Arc," and "nuclear Arc," respectively (Fig. 3C,D). We measured the proportions of active neurons in the BLA during sessions 1, 2, and 3 (Fig. 3D). There was no group effect or group $\times$ session interaction (Fig. $3 E$ ).
A

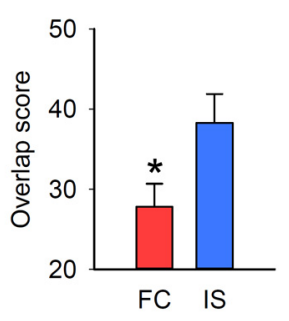

B

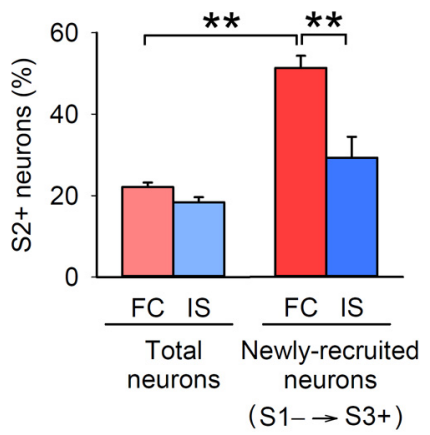

C

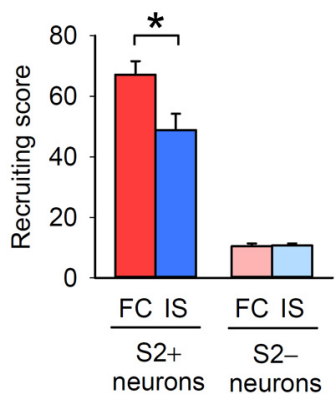

D

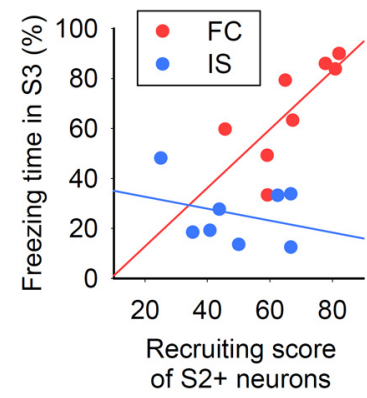

Figure 4. Fear conditioning alters a context-responsive BLA neuronal ensemble based on the neuronal activity during fear conditioning. $\boldsymbol{A}$, BLA neurons that were active in session 3 overlapped less with those that were active in session 1 in the FC group compared with the IS group (Student's $t$ test, $t_{(14)}=2.3, p=0.039$ ). $B$, BLA neurons newly recruited into the context-responsive ensemble displayed preferential activity in session 2 depending on fear learning (repeated-measures ANOVA, $F_{(1,14)}=11.0, p=0.0051$; total vs newly recruited neurons in the $\mathrm{F}\left(\right.$ group, $\left.p=1.5 \times 10^{-5}\right)$. $C$, The activities of individual BLA neurons during fear conditioning predicted their recruitment into the context-responsive ensemble $\left(F_{(1,14)}=\right.$ $7.08, p=0.019 ; \mathrm{S} 2+(\mathrm{FC})$ vs $\mathrm{S} 2-(\mathrm{FC}), p=0.00062 ; \mathrm{S} 2+(\mathrm{FC})$ vs $\mathrm{S} 2+(\mathrm{IS}), p=0.021) . \boldsymbol{D}$, The recruiting effect of activity during fear conditioning in the $B L A$ correlated with the strength of fear memory expression (Pearson's correlation coefficient, $r=0.74, t_{(7)}=2.7, p=0.036$; Z-test, FC vs IS, $z=2.0, p=0.023) ;{ }^{* *} p<0.01,{ }^{*} p<0.05$.

To determine whether fear conditioning alters the contextresponsive neuronal ensemble, the overlap between the neuronal populations that were active during sessions 1 and 3 was examined. The overlap score of the FC group was lower than that of the IS group (Fig. 4A). This result indicates that fear conditioning alters the context-responsive neuronal ensemble.

We further hypothesized that the newly recruited neurons were preferentially activated during conditioning, because synaptic potentiation was observed in neurons that were activated during conditioning. To test this, we measured the percentage of neurons that were active during session $2(\mathrm{~S} 2+)$ among the total BLA neurons and among the newly recruited neurons. In the FC group, the $\mathrm{S} 2+$ percentage in the newly recruited neurons was higher than that in the total BLA neurons (Fig. $4 B$ ). In the IS group, on the other hand, there was no significant difference.

To determine whether neurons activated during fear conditioning are newly recruited into the context-responsive ensemble, we calculated a recruiting score consisting of the percentage of $\mathrm{S} 2+$ or $\mathrm{S} 2-$ neurons that changed their activity from inactive in session 1 to active in session 3. In the FC group, the recruiting score of the S2+ neurons was higher than that of the S2- neurons (Fig. 4C). Further analysis also indicated that the recruiting effect of session 2 activity was associated with fear conditioning: First, the recruiting score of S2+ neurons of the FC group was higher than that of the IS group (Fig. 4C); second, the recruiting 
score in the FC group correlated positively with the freezing time in session 3 (Fig. 4D). Together, these results indicate that fear conditioning alters the context-responsive neuronal ensemble depending on the neuronal activity during fear conditioning.

\section{Discussion}

We demonstrated that contextual fear conditioning induces presynaptic potentiation in cortical, but not thalamic, synapses on BLA neurons that were recruited into the fear memory trace. In accordance with the hypothesis that synaptic modification in a subset of neurons is likely to lead to a reorganization of neuronal ensembles, we also found that fear conditioning alters a contextresponsive neuronal ensemble depending on the neuronal activity during fear conditioning. The recruiting effect of activity during fear conditioning correlated with the strength of fear memory expression. Overall, these data suggest that synaptic plasticity in a subset of BLA neurons is likely to contribute to fear memory expression through ensemble reorganization.

A novel finding of this study is that synaptic potentiation associated with learning occurs specifically in neurons that are recruited into the memory trace. Although previous studies showed that fear conditioning induces synaptic potentiation in the BLA synapses (McKernan and Shinnick-Gallagher, 1997; Tsvetkov et al., 2002), it is unclear whether fear conditioning induces synaptic modification of the amygdala globally or only in a subset of neurons. In this study, we challenged this issue by measuring synaptic efficacy from neurons recruited into a memory trace, which are labeled with $A r c$ promoter-driven dVenus. We found that presynaptic potentiation is specifically induced in the recruited neurons (dVenus + neurons). The observed increase in mEPSC frequency in dVenus + neurons from conditioned mice reflects modifications in whole neurons, whereas the decrease in the PPR was limited to the cortical pathway alone. This might be due to the greater influence of cortical inputs on BLA neurons relative to other inputs. Our findings do not rule out postsynaptic plasticity (Rumpel et al., 2005; Zhou et al., 2009) as another mechanism for contextual fear conditioning. The amplitude of mEPSCs in the dVenus + neurons was higher compared with dVenus - neurons, but there was no group $\times d$ Venus interaction. The postsynaptic enhancement effect of fear conditioning might be masked by footshock stress, which was also given to the IS group.

The activity of individual neurons during learning is likely to determine their recruitment into the memory trace. In this study, we used large-scale activity mapping with the immediate-early genes Arc and Homer $1 a$ to determine how contextual fear conditioning alters BLA neuronal ensembles. Remarkably, we measured the activities of $>300$ neurons per mouse separately over three sessions by analyzing subcellular distributions of Arc and Homer $1 a$ RNA. In doing so, we found that the neurons activated during memory retrieval were preferentially activated during preceding fear conditioning, and the neurons that were activated during fear conditioning were also preferentially activated during memory retrieval. The importance of neuronal activity during learning in recruitment is supported by previous studies using CREB transfection. CREB transfection upregulates excitability of transfected neurons (Zhou et al., 2009) and increases the recruitment ratio of those neurons into a fear memory trace (Han et al., 2007).Together, these findings suggest that the recruitment of neurons into a memory trace is defined by the activity of individual neurons during learning.

In conclusion, we have demonstrated that presynaptic potentiation occurs specifically in neurons that are recruited into a fear memory trace. This synaptic plasticity likely contributes to the ensemble reorganization, an event that positively correlated with the strength of fear memory expression. Together, our findings suggest that synaptic plasticity in a subset of BLA neurons contributes to fear memory expression through the reorganization of a context-responsive neuronal ensemble.

\section{References}

Eguchi M, Yamaguchi S (2009) In vivo and in vitro visualization of gene expression dynamics over extensive areas of the brain. Neuroimage 44: 1274-1283. CrossRef Medline

Fanselow MS, Kim JJ (1994) Acquisition of contextual Pavlovian fear conditioning is blocked by application of an NMDA receptor antagonist D, L-2-amino-5-phosphonovaleric acid to the basolateral amygdala. Behav Neurosci 108:210-212. CrossRef Medline

Guzowski JF, McNaughton BL, Barnes CA, Worley PF (1999) Environmentspecific expression of the immediate-early gene Arc in hippocampal neuronal ensembles. Nat Neurosci 2:1120-1124. CrossRef Medline

Han JH, Kushner SA, Yiu AP, Cole CJ, Matynia A, Brown RA, Neve RL, Guzowski JF, Silva AJ, Josselyn SA (2007) Neuronal competition and selection during memory formation. Science 316:457-460. CrossRef Medline

Han JH, Kushner SA, Yiu AP, Hsiang HL, Buch T, Waisman A, Bontempi B, Neve RL, Frankland PW, Josselyn SA (2009) Selective erasure of a fear memory. Science 323:1492-1496. CrossRef Medline

Hashikawa K, Matsuki N, Nomura H (2011) Preferential arc transcription at rest in the active ensemble during associative learning. Neurobiol Learn Mem 95:498-504. CrossRef Medline

Li XB, Inoue T, Nakagawa S, Koyama T (2004) Effect of mediodorsal thalamic nucleus lesion on contextual fear conditioning in rats. Brain Res 1008:261-272. CrossRef Medline

Maren S (2001) Neurobiology of Pavlovian fear conditioning. Annu Rev Neurosci 24:897-931. CrossRef Medline

Maren S, Fanselow MS (1997) Electrolytic lesions of the fimbria/fornix, dorsal hippocampus, or entorhinal cortex produce anterograde deficits in contextual fear conditioning in rats. Neurobiol Learn Mem 67:142-149. CrossRef Medline

Marrone DF, Schaner MJ, McNaughton BL, Worley PF, Barnes CA (2008) Immediate-early gene expression at rest recapitulates recent experience. J Neurosci 28:1030-1033. CrossRef Medline

McKernan MG, Shinnick-Gallagher P (1997) Fear conditioning induces a lasting potentiation of synaptic currents in vitro. Nature 390:607-611. CrossRef Medline

Miura Y, Naka M, Matsuki N, Nomura H (2012) Differential calcium dependence in basal and forskolin-potentiated spontaneous transmitter release in basolateral amygdala neurons. Neurosci Lett 529:1-6. CrossRef Medline

Nomura H, Matsuki N (2008) Ethanol enhances reactivated fear memories. Neuropsychopharmacology 33:2912-2921. CrossRef Medline

Nomura H, Nonaka A, Imamura N, Hashikawa K, Matsuki N (2012) Memory coding in plastic neuronal subpopulations within the amygdala. Neuroimage 60:153-161. CrossRef Medline

Reijmers LG, Perkins BL, Matsuo N, Mayford M (2007) Localization of a stable neural correlate of associative memory. Science 317:1230-1233. CrossRef Medline

Rumpel S, LeDoux J, Zador A, Malinow R (2005) Postsynaptic receptor trafficking underlying a form of associative learning. Science 308:83-88. CrossRef Medline

Sacchetti B, Lorenzini CA, Baldi E, Tassoni G, Bucherelli C (1999) Auditory thalamus, dorsal hippocampus, basolateral amygdala, and perirhinal cortex role in the consolidation of conditioned freezing to context and to acoustic conditioned stimulus in the rat. J Neurosci 19:9570-9578. Medline

Tsvetkov E, Carlezon WA, Benes FM, Kandel ER, Bolshakov VY (2002) Fear conditioning occludes LTP-induced presynaptic enhancement of synaptic transmission in the cortical pathway to the lateral amygdala. Neuron 34:289-300. CrossRef Medline

Zhou Y, Won J, Karlsson MG, Zhou M, Rogerson T, Balaji J, Neve R, Poirazi P, Silva AJ (2009) CREB regulates excitability and the allocation of memory to subsets of neurons in the amygdala. Nat Neurosci 12:14381443. CrossRef Medline 